\title{
Mediators of extracellular matrix degradation and inflammation: A new team of possible biomarkers for oral squamous cell carcinoma stage
}

\author{
RADU RADULESCU ${ }^{1 *}$, ALEXANDRA RIPSZKY TOTAN $^{1 *}$, MARINA MELESCANU IMRE $^{2 *}$, \\ DANIELA MIRICESCU ${ }^{1}$, ANDREEA DIDILESCU ${ }^{3}$ and MARIA GREABU ${ }^{1}$
}

\author{
${ }^{1}$ Department of Biochemistry, Faculty of Dental Medicine, 'Carol Davila' University of Medicine and Pharmacy, \\ 050474 Bucharest; ${ }^{2}$ Department of Complete Denture, Faculty of Dental Medicine, 'Carol Davila' \\ University of Medicine and Pharmacy, 032799 Bucharest; ${ }^{3}$ Department of Embryology, Faculty of Dental Medicine, \\ 'Carol Davila' University of Medicine and Pharmacy, 050474 Bucharest, Romania
}

Received December 24, 2020; Accepted January 26, 2021

DOI: $10.3892 / \mathrm{etm} .2021 .10309$

\begin{abstract}
Oral cancer represents one of the most common types of cancer worldwide, with oral squamous cell carcinoma (OSCC) being the most frequently diagnosed. Cytokines play a crucial role in inflammation, apoptosis and metastasis. Interleukin (IL)-8 promotes the direct migration of inflammatory cells. IL-6 induces tumor cell proliferation, increases expression of invasiveness and angiogenetic factors or matrix metalloproteinases (MMPs), promoting metastasis. Tissue inhibitor of metalloproteinases (TIMPs) blocks the action of MMPs controlling extracellular matrix degradation and inhibiting metastasis. The aim of our study was to analyze the existence of correlations between inflammation markers (IL-6 and IL-8) and extracellular degradation protection markers such as TIMP-1 in OSCC tumors. Our study included 20 patients (12 females and 8 males) diagnosed with OSCC, recruited from January to April, 2020. IL-8, IL-6 and TIMP-1 levels were measured in the tumor cell lysates by ELISA technique, using relevant assay kits. Our results showed a positive and significant correlation between IL-6 and IL-8 ( $\mathrm{P}=0.005$, $\mathrm{R}=0.517$ ) indicating that high IL- 8 levels can be associated
\end{abstract}

Correspondence to: Professor Alexandra Ripszky Totan, Department of Biochemistry, Faculty of Dental Medicine, 'Carol Davila' University of Medicine and Pharmacy; 8 Eroii Sanitari Boulevard, 050474 Bucharest, Romania

E-mail: alexandra.totan@umfcd.ro

Professor Marina Melescanu Imre, Department of Complete Denture, Faculty of Dental Medicine, 'Carol Davila' University of Medicine and Pharmacy, 8 Aleea Barajul Iezeru, 032799 Bucharest, Romania

E-mail: marina.imre@umfcd.ro

*Contributed equally

Key words: inflammation, cellular matrix degradation, oral cavity, squamous cell carcinoma, IL-6, IL-8, TIMP-1, MMP with high IL-6 levels. We also found a significant and high negative correlation $(\mathrm{P}<0.001, \mathrm{R}=-0.673)$ between IL-6 and TIMP-1 and a significant and high negative correlation $(\mathrm{P}<0.001, \mathrm{R}=-0.684)$ between IL-8 and TIMP-1 indicating that high levels of IL-8 and IL-6 are significantly associated with lower levels of TIMP-1. In conclusion, our study confirms the available literature data on IL- 6 and IL- 8 as potential markers for oral cancers such as OSCC and affect the tumor microenvironment by decreasing TIMPs. All three biomarkers included in this study have the potential to be used as detection or prognostic factors for oral cancer.

\section{Introduction}

Oral cancer represents one of the most frequent types of cancer worldwide with an incidence of about $9 \%$ of all types or cancer (1). Oral malignant tumors differ from benign tumors by their rate of high invasiveness accompanied by local tissue destruction and a high lymphatic and circulatory metastasis rate (2).

The most common sites for oral cancer are the lower lip, the tongue and the mouth floor mucosa. Usually older patients are most susceptible for developing oral cancers; individuals between 50 and 55 years of age having the highest incidence (3).

Varied clinical and histological forms of oral malignant lesions have been described, oral squamous cell carcinoma (OSCC) being the most frequently diagnosed form, comprising $\sim 90 \%$ of all oral cancers (4). This type of cancer is characterized by a high mortality rate of over $50 \%$ due to its high invasiveness, chemotherapy and radiotherapy resistance and late stage diagnosis mostly in the inoperable state $(5,6)$. One key factor in lowering oral cancer morbidity is an early stage diagnosis which greatly improves long term survivability (7).

Early stage diagnosis can be achieved by combining clinical detection methods such as vital staining, brush biopsy, auto-fluorescence spectroscopy, chemiluminescent illumination, narrow band imaging and confocal microscopy (8). These methods are invasive and can detect emergent oral cancer only after dysplastic changes have occurred (9). 
Combining clinical methods with laboratory testing in which key biomarkers in early stage cancer development could be analyzed and used to predict the existence of malignant transformation or to evaluate the prognosis in late stage diagnose of oral neoplasms is crucial. These key biomarkers could be analyzed either in serum, saliva or tissue samples.

Cancer research has identified more than one hundred biomarkers that show a potential for a laboratory diagnosis of oral cancers. These biomarkers can be specific for each stage and mechanism responsible for the initiation, proliferation and metastasis of oral cancers $(10,11)$.

From these potential biomarkers, in the present study we include key inflammatory cytokines such as interleukin (IL)-6 and IL-8 and tissue inhibitor of metalloproteinase-1 (TIMP-1), a protective factor against extracellular matrix degradation.

Cytokines are a group of low-molecular-weight glycoproteins produced by immune and non-immune cells and play a crucial role as molecular messengers in inflammation, apoptosis, and host resistance acting as signaling molecules in most cellular interactions (12).

Key characteristics of cytokines are the ability to interact only with cellular targets and not between themselves and that one cell can respond to several cytokines (9).

IL-8 is a pro-inflammatory cytokine released by various inflammatory cells such as neutrophils and macrophages following the nuclear factor $-\kappa \mathrm{B}(\mathrm{NF}-\kappa \mathrm{B})$ pathway activation by various stimuli such as inflammatory signals and environmental stresses (13). IL-8 acts on two cellular receptors CRCX-1 and CRCX-2 which are structurally similar, found on the surface of macrophages, neutrophils and most important on the surface of cancer cells (14).

The traditional role of IL-8 is to promote direct migration of inflammatory cells following a concentration gradient leading to an accumulation at the site of IL- 8 production and to facilitate the degranulation of neutrophils (13). Cancer cells can obtain the ability to release different cytokines such as IL-8 which can ultimately lead to metastasis by promoting neutrophil recruitment, angiogenesis, proliferation of endothelial cells and apoptosis resistance (15). IL- 8 binding to CRCX-1 and CRCX-2 receptors triggers the activation of several downstream signaling pathways such as the phosphatidylinositol-3 kinase (PI3K)/Akt, mitogen-activated protein kinase (MAPK) and Janus kinase (JAK)/signal transducer and activator of transcription (STAT) pathway $(14,16)$.

IL-6 is a pro-inflammatory cytokine released by various cells such as immune cells that infiltrate the tumor such as macrophages, a wide range of stromal cells and also by the tumor cells themselves, activating JAK/STAT3 and MAPK pathways leading to encoding of proteins that induce tumor cell proliferation (cyclin D1) and survival (BCL2-like protein) (17). STAT3 activation also leads to increased expression of invasiveness and angiogenetic factors such as vascular endothelial growth factor (VEGF) or matrix metalloproteinases (MMPs) thus promoting metastasis (18). Another effect of JAK/STAT3 activation by IL-6 is the resulting immunosuppressive tumor microenvironment due to the IL-6 inhibition of neutrophils, natural killer cells and $\mathrm{T}$ cells leading to a decrease in antitumor immunity (19).

Extracellular matrix (ECM) macromolecules create the environment required for normal tissue function, including the precise regulation of formation and degradation. Key enzymes in ECM degradation include the matrix metalloproteinases (MMPs), a group of proteolytic enzymes consisting of a prodomain, a catalytic region, a hinge region and a hemopexin domain and are secreted by both normal and cancer cells $(20,21)$.

TIMPs are a group of four proteins that block the action of various classes of MMPs by inhibiting the catalytic domain of MMPs based on the interactions between the $\mathrm{N}$-terminal domain of TIMPs which is structurally similar with the substrate of MMPs (22). Recent studies have linked TIMPs with other inhibitory roles on non-MMP metalloproteinase such as the metallopeptidases ADAMs and ADAMTS (23). TIMPs also exhibit cell growth-promoting roles; overexpression of some types of TIMPs reduces tumor growth (20).

The aim of the present study was to analyze the existence of various correlations between inflammation markers (IL-6 and IL-8) and extracellular matrix degradation protection markers such as TIMP-1 in OSCC tumors.

\section{Patients and methods}

Our study included 20 patients (12 females and 8 males) diagnosed with oral squamous cell carcinoma, recruited from January to April 2020 from the patients treated at the Oro-Maxillo-Facial Hospital 'Prof. Dr. Dan Theodorescu' Bucharest. Patients age included in this study ranged from 36 to 75 years, the mean age being $55.0 \pm 10.9$ years (mean \pm SD). Ten patients were active smokers. Only one patient ceased smoking before inclusion in this study and nine were nonsmokers.

Our prospective study was initiated following approval of the Ethics Committee of UMF Carol Davila. Informed written consent was obtained from all individual participants included in the study. Oral cancer tissue samples were collected from the included participants during surgery. Tumor cell lysates were obtained according to the assay kit manufacturer's recommendations.

IL-8, IL-6 and TIMP-1 levels were measured in the tumor cell lysates by ELISA technique, using assay kits (cat. nos. E-EL-H0048, E-EL-H0102 and E-EL-H0184) from Elabscience numbers.

Statistical analysis. The results were statistically analyzed using IBM SPSS Statistics 25 (IBM Corp.), Microsoft Office Excel/Word 2013 and the Shapiro-Wilk distribution test. For correlation analysis, the Spearman's rho correlation coefficient was used.

\section{Results}

In the present study, possible correlations were analyzed between inflammation markers IL- 6 and IL- 8 and TIMP-1, a protective marker against degradation of the ECM.

Our results presented in Table I and Fig. 1 revealed a non-parametric distribution, according to Shapiro-Wilk test $(\mathrm{P}<0.05)$ between IL-8 and IL-6 inflammation markers. A positive and significant correlation between IL-6 and IL-8 was also observed $(\mathrm{P}=0.005, \mathrm{R}=0.517)$ indicating that high IL-8 levels can be associated with a significantly higher frequency of high IL-6 levels. 
Table I. Correlation between IL-8 and IL-6 levels.

Correlation P-value

IL-8 $(\mathrm{P}<0.001) \times$ IL-6 $(\mathrm{P}<0.001)$

$0.005, \mathrm{R}=0.517$

IL, interleukin.

Table II. Correlation between IL-6 and TIMP-1 .

\begin{tabular}{lc}
\hline Correlation & P-value \\
\hline TIMP-1 $(\mathrm{P}=0.005) \times$ IL-6 $(\mathrm{P}<0.001)$ & $<0.001, \mathrm{R}=-0.673$ \\
\hline
\end{tabular}

IL, interleukin; TIMP-1, tissue inhibitor of metalloproteinase-1.

Table III. Correlation between IL- 8 and TIMP-1.

\begin{tabular}{lc}
\hline Correlation & P-value \\
\hline TIMP-1 $(\mathrm{P}=0.005) \times$ IL-8 $(\mathrm{P}<0.001)$ & $<0.001, \mathrm{R}=-0.684$
\end{tabular}

IL, interleukin; TIMP-1, tissue inhibitor of metalloproteinase-1.

The next correlation analyzed was between IL-6 and TIMP-1 and the results are presented in Table II and Fig. 2. A non-parametric distribution is revealed, according to Shapiro-Wilk test $(\mathrm{P}<0.05)$ and the correlation between the studied parameters showed a significant and high degree negative correlation $(\mathrm{P}<0.001, \mathrm{R}=-0.673)$ indicating that high levels of IL-6 are significantly associated with lower levels of TIMP-1.

The final correlation to be analyzed was between IL- 8 and TIMP-1 and the results are presented in Table III and Fig. 3. A non-parametric distribution is revealed, according to Shapiro-Wilk test $(\mathrm{P}<0.05)$ and the correlation between the studied parameters showed significant and a high negative correlation $(\mathrm{P}<0.001, \mathrm{R}=-0.684)$ indicating that high levels of IL-8 are significantly associated with lower levels of TIMP-1.

\section{Discussion}

Inflammation represents the response to various tissue aggressions caused by physical trauma, ischemic conditions, infections or extended exposure to toxic agents resulting in an inflammatory response aimed at repairing the damage consisting of cellular transformation and proliferation (24). In the case of persistent tissue damage or when control mechanisms are altered or incapacitated, acute inflammation can result in a chronic state of inflammation characterized by high mutation and cellular proliferation rates creating conditions for the onset of cancer (25).

After initial malignant cell transformation, cancer progression, according to Hanahan and Weinberg is based on 10 key elements such as: Sustained proliferative signals, evasion of cellular growth suppressors, metastasis through local invasion, promotion of inflammation, immortality, induction of angiogenesis, genetic instability and mutations, cellular death resistance and metabolic imbalances (26).

It is widely accepted that chronic inflammation is responsible for almost a quarter of all malignancies diagnosed (27). Chronic inflammation can play a crucial role in cancer progression acting on many of the key inflammatory steps such as cellular proliferation, local invasiveness, angiogenesis, metastasis, and cytokines such as IL-1, IL-6, IL-8 and tumor necrosis factor (TNF)- $\alpha$ representing key elements (28).

A wide number of both normal or cancer cells have the ability to release IL- 8 after the activation of nuclear factor $(\mathrm{NF})-\kappa \mathrm{B}$ as a response to various local and systemic stimuli (9). In normal unstimulated cells, IL-8 levels are virtually undetected. Apart from $\mathrm{NF}-\kappa \mathrm{B}$ activation pathway, de-repression of the IL-8 gene promoter and IL-8 mRNA stabilization by the p38 MAPK pathway play crucial roles in IL-8 release (29).

IL-8 has an autocrine and paracrine tumor-promoting role, altering the local microenvironment, inducing cell growth mainly in endothelial cells, stimulating leukocyte infiltration and modification of immune responses (30).

IL-8 binds with high specificity with two membrane receptors CXCR1 and CXCR2 located on tumor-associated macrophages, neutrophils and cancer cells. This binding activates protein-G mediated pathways leading to calcium release and activation of the Ras/MAPK and PI3K signaling cascades (29). CXCR1 and not CXCR2 activates phospholipase $\mathrm{D}$ leading to an increased oxidative burst through increased reactive oxygen species production contributing to altered metabolic conditions and leading to further mutations in the tumor microenvironment $(31,32)$. The angiogenesis effect of IL- 8 can be explained by the binding of IL- 8 to CXCR receptors that triggers the increase in Bcl-2 expression and matrix metalloproteinase (MMP) production, through ERK phosphorylation, leading to endothelial cell proliferation and a degradation of the extracellular matrix (ECM) which creates the local conditions for proliferation $(33,34)$.

Cellular proliferation and survival effects on other types of cells of IL-8 can be explained through the activation of Src-kinases and focal adhesion kinase (FAK) by increasing phosphorylation $(35,36)$.

The other pro-inflammatory cytokine included in this study is IL-6. It plays important roles in cellular proliferation, angiogenesis, local invasion, regenerative and metabolic processes. It also regulates cellular metabolism protecting cancer cells from the hypoxic conditions that characterize the tumor microenvironment (9).

A host of cells located at the tumor microclimate level secrete IL-6, including inflammatory cells, normal stromal cells and also cancer cells. The effects of IL- 6 are manifested at both the local and systemic levels $(37,38)$.

Cellular and metabolic actions of IL- 6 can be mediated through the JAK/STAT3 and Ras/Raf/MAPK pathways $(9,18)$. JAK/STAT3 pathway activation by IL-6 leads to the stimulation of target genes, such as cyclin D1 responsible for essential roles in the $\mathrm{G} 1$ phase of the cell cycle, the stimulation of $\mathrm{Bcl}-2$ protein affecting the regulatory mechanisms of apoptosis, the 


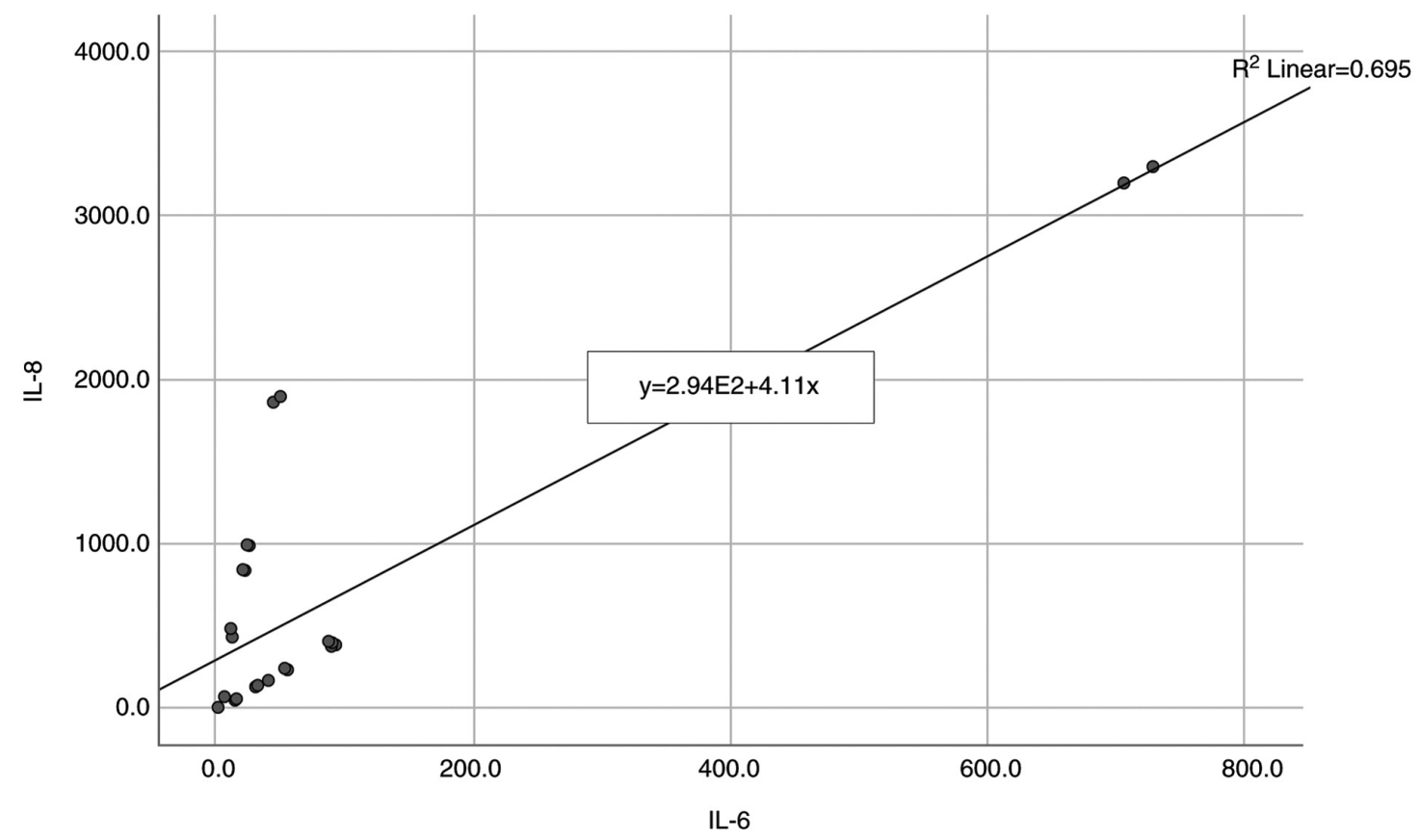

Figure 1. Positive and significant correlation between IL-8 and IL-6 levels. IL, interleukin.

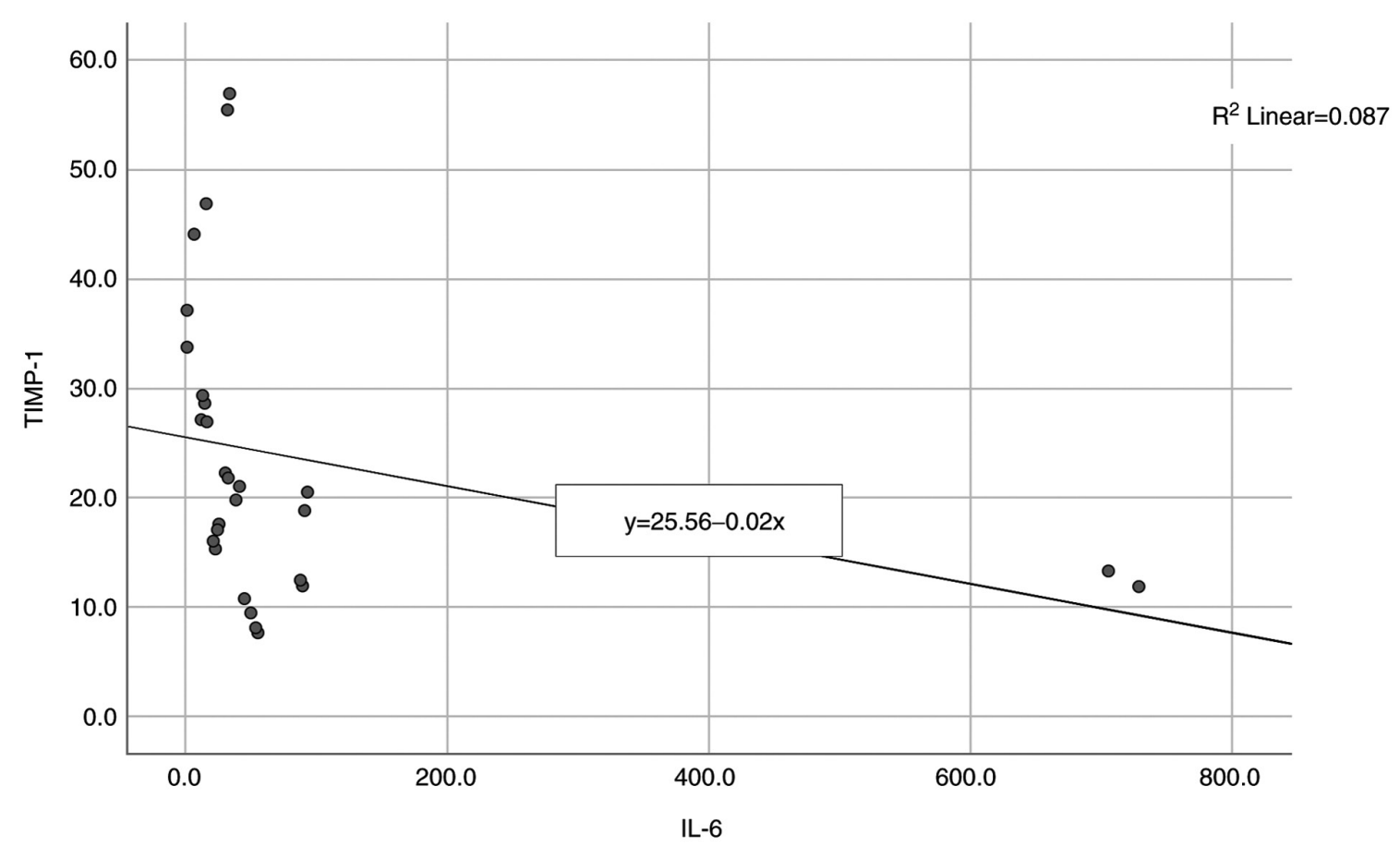

Figure 2. A negative and significant correlation between IL-6 and TIMP-1. IL, interleukin; TIMP-1, tissue inhibitor of metalloproteinase-1.

release of angiogenesis-stimulating factors such as vascular endothelial growth factor (VEGF) or increased levels of local invasiveness markers such as MMPs $(18,39,40)$. Another effect of IL-6, coupled with high levels of oxidative stress is the enhanced methylation of tumor-suppressor genes and microRNAs through DNA methyltransferase 1 (DNMT1) transcription alteration (41).

The release of IL- 6 can be further increased by the binding of STAT3 to the promotor sequence of IL- 6 release establishing a positive feedback loop increasing the biological effects (42).
Local invasiveness is another key mechanism in oral cancer progression. MMPs are a group of zinc-dependent endoproteases with roles in degradation and remodeling of the ECM and are secreted by both normal and tumor cells (43). They act on all types of collagen and elastin in the ECM, hemopexin domain conferring substrate specificity for different collagen types (44). High levels of MMPs, especially MMP-9, were found in low to moderate differentiated tumors and were found to be secreted by cancer and inflammatory cells such as macrophages both in the primary tumor, mostly 


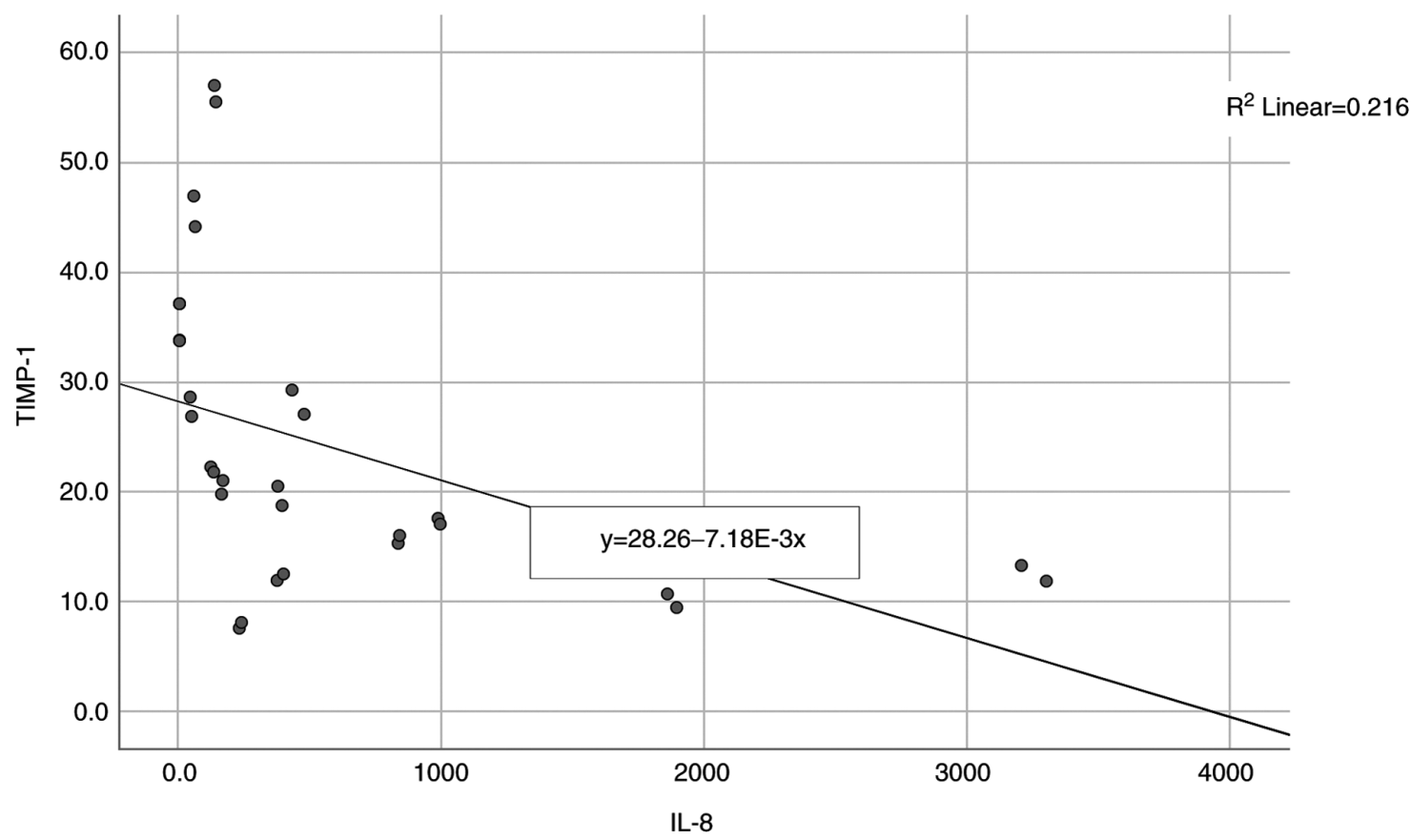

Figure 3. A significant negative correlation between IL-8 and TIMP-1. IL, interleukin; TIMP-1, tissue inhibitor of metalloproteinase-1.

at the tumor invasion margins, or in lymph node metastasis inducing angiogenesis thus leading to invasiveness and cell growth (45). MMP expression is regulated at the transcription level by cytokines, at pro-enzyme activation level by oxidative stress and by the TIMP concentration (46).

The direct inhibitors and regulators of MMPs are TIMPs. Any disruption in the balance between MMP activity and TIMP inhibition may lead to invasion and metastasis and a worse prognosis $(47,48)$.

Lower levels of TIMP-1 can be explained by the alteration in phosphorylation of key MAPK pathway molecules such as JNK, Erk and p38 following miR-196 activation leading to suppressed TIMP-1 levels and elevated MMP levels (49).

Many studies have analyzed either IL- 8 or IL- 6 levels separately or together with other biomarkers using either saliva, serum or tumors as samples. Elevated levels of IL-6 and IL- 8 were found by St John et al both in the serum and saliva of oral squamous cell carcinoma (OSCC) patients, IL-8 had higher concentrations in saliva samples and IL-6 had higher concentrations in serum (50). These results were confirmed by SahebJamee et al using only saliva as a diagnostic fluid (51). Punyani and Sathawane found elevated levels of IL-8 in both premalignant and OSCC cancer patients (52). Higher levels of IL-6 in OSCC patients were found by Lotfi et al (53). A meta-analysis of 24 studies conducted by Rezaei et al concluded that IL-8 and IL-6 levels were statistically higher in OSCC patients (54).

The diagnostic value of IL- 8 levels could be influenced by other diseases in which high levels of IL- 8 are found, such as asthma both allergic and non-allergic types, or other common inflammatory diseases, such as viral infections (55).

In the present study, both chronic inflammation markers, IL-8 and IL-6, were present in high concentrations in OSCC cell lysates and after statistical analysis we found a positive and significant correlation between these two parameters
$(\mathrm{P}=0.005, \mathrm{R}=0.517)$ indicating that high levels of IL-8 can be associated with high levels of IL-6. A high concentration and correlation between these biomarkers can suggest that both OSCC cells and normal cells surrounding the tumor actively secrete cytokines. A high concentration of IL-8 in the tumor microenvironment can be seen as a defensive measure in order to attract more inflammatory cells. IL-8 and IL- 6 can also play a negative role in cancer progression inducing angiogenesis, cellular proliferation and cancer cell survival.

ECM degradation marker analyzed in this study was TIMP-1. The level of this parameter was decreased in OSCC and after statistical analysis, a negative and significant correlation between IL-8 and TIMP-1 ( $\mathrm{P}<0.001, \mathrm{R}=-0.684)$ and between IL-6 and TIMP 1 was found $(\mathrm{P}<0.001, \mathrm{R}=-0.673)$ which indicates that high levels of IL- 6 or IL- 8 can be statistically correlated with low levels of TIMP-1 in OSCC patients. To the best of our knowledge, this is the first study where the statistical correlation between TIMP-1 and IL- 6 and IL-8 was conducted.

The possible connection between IL- 6 and IL- 8 and TIMP-1 can be explained by the inhibitory effect of these cytokines on microRNA expression, which can control via MAPK pathways the production of TIMPs (18). We can speculate that another consequence of high cytokine expression in cancers and in oral cancer in particular is the inhibition of ECM degradation control mechanisms.

In conclusion, our study confirms the available literature data on IL- 6 and IL- 8 as potential markers for oral cancers such as OSCC. The negative correlations between IL-6 and TIMP-1 and IL-8 and TIMP-1 suggest that pro-inflammatory cytokines affect the tumor microenvironment by decreasing MMP control factors such as TIMPs. All three biomarkers included in this study have the potential to be used as detection or prognostic factors for oral cancer. 


\section{Acknowledgements}

Not applicable.

\section{Funding}

This paper was financially supported by 'Carol Davila' University of Medicine and Pharmacy (contract no. 23PFE/17.10.2018) funded by the Ministry of Research and Innovation within PNCDI III, Program 1-Development of the National R\&D system, Subprogram 1.2-Institutional Performance-RDI excellence funding projects.

\section{Availability of data and materials}

All data generated or analyzed during the present study are included in this published article.

\section{Authors' contributions}

RR, AD, MMI, ART, DM and MG made substantial contributions to the conception and design of the research. ART, $\mathrm{RR}, \mathrm{AD}, \mathrm{DM}$ and MMI made substantial contributions to the acquisition, analysis, and interpretation of data for the research. RR, ART and MMI drafted the work and revising it critically for important intellectual content. AD and MG gave final approval of the version to be published. All authors read and approved the manuscript and agree to be accountable for all aspects of the research in ensuring that the accuracy or integrity of any part of the work are appropriately investigated and resolved.

\section{Ethics approval and consent to participate}

Our prospective study was initiated after the approval of the Ethics Committee of 'Carol Davila' University of Medicine and Pharmacy, no. 32698/11.12.2020. Informed written consent was obtained from all individual participants included in the study.

\section{Patient consent for publication}

Not applicable.

\section{Competing interests}

The authors declare that they have no competing interests.

\section{References}

1. Ghantous Y and Abu Elnaaj I: Global incidence and risk factors of oral cancer (In Hebrew). Harefuah 156: 645-649, 2017.

2. Rivera C: Essentials of oral cancer. Int J Clin Exp Pathol 8: 11884-11894, 2015.

3. Liu D, Yang F, Xiong F and Gu N: The smart drug delivery system and its clinical potential. Theranostics 6: 1306-1323, 2016.

4. Hema KN, Smitha T, Sheethal HS and Mirnalini SA: Epigenetics in oral squamous cell carcinoma. J Oral Maxillofac Pathol 21: 252-259, 2017.

5. Wong T and Wiesenfeld D: Oral cancer. Aust Dent J 63 (Suppl 1): S91-S99, 2018

6. Bais MV: Impact of epigenetic regulation on head and neck squamous cell carcinoma. J Dent Res 98: 268-276, 2019.
7. Scully $\mathrm{C}$ and Kirby J: Statement on mouth cancer diagnosis and prevention. Br Dent J 216: 37-38, 2014.

8. Kalavrezos N and Scully C: Mouth cancer for clinicians part 7: Cancer diagnosis and pre-treatment preparation. Dent Update 43: 50-54, 57-60, 63-65, 2016.

9. Sahibzada HA, Khurshid Z, Khan RS, Naseem M, Siddique KM Mali M and Zafar MS: Salivary IL-8, IL-6 and TNF- $\alpha$ as potential diagnostic biomarkers for oral cancer. Diagnostics (Basel) 7: 21, 2017.

10. Cheng YS, Rees T and Wright J: A review of research on salivary biomarkers for oral cancer detection. Clin Transl Med 3: 3, 2014.

11. Hussein AA, Forouzanfar T, Bloemena E, de Visscher J, Brakenhoff RH, Leemans CR and Helder MN: A review of the most promising biomarkers for early diagnosis and prognosis prediction of tongue squamous cell carcinoma. Br J Cancer 119: 724-736, 2018.

12. Lee $\mathrm{S}$ and Margolin K: Cytokines in cancer immunotherapy. Cancers (Basel) 3: 3856-3893, 2011.

13. David JM, Dominguez C, Hamilton DH and Palena C: The IL-8/IL-8R axis: A double agent in tumor immune resistance. Vaccines (Basel) 4: 22, 2016.

14. Waugh DJ and Wilson C: The interleukin-8 pathway in cancer. Clin Cancer Res 14: 6735-6341, 2008.

15. De Larco JE, Wuertz BR and Furcht LT: The potential role of neutrophils in promoting the metastatic phenotype of tumors releasing interleukin-8. Clin Cancer Res 10: 4895-4900, 2004.

16. Long X, Ye Y, Zhang L, Liu P, Yu W, Wei F, Ren X and Yu J: IL-8, a novel messenger to cross-link inflammation and tumor EMT via autocrine and paracrine pathways (Review). Int J Oncol 48: 5-12, 2016.

17. Kumari N, Dwarakanath BS, Das A and Bhatt AN: Role of interleukin-6 in cancer progression and therapeutic resistance. Tumour Biol 37: 11553-11572, 2016.

18. Johnson DE, O'Keefe RA and Grandis JR: Targeting the IL-6/JAK/STAT3 signalling axis in cancer. Nat Rev Clin Oncol 15: 234-248, 2018.

19. Lee H, Pal SK, Reckamp K, Figlin RA and Yu H: STAT3: A target to enhance antitumor immune response. Curr Top Microbiol Immunol 344: 41-59, 2011.

20. Visse R and Nagase H: Matrix metalloproteinases and tissue inhibitors of metalloproteinases: Structure, function, and biochemistry. Circ Res 92: 827-839, 2003.

21. Cui N, Hu M and Khalil RA: Biochemical and biological attributes of matrix metalloproteinases. Prog Mol Biol Transl Sci 147: 1-73, 2017

22. Arpino V,Brock M and Gill SE: The role of TIMPs in regulation of extracellular matrix proteolysis. Matrix Biol 44-46: 247-254, 2015.

23. Brew $\mathrm{K}$ and Nagase $\mathrm{H}$ : The tissue inhibitors of metalloproteinases (TIMPs): An ancient family with structural and functional diversity. Biochim Biophys Acta 1803: 55-71, 2010.

24. Coussens LM and Werb Z: Inflammation and cancer. Nature 420 : 860-867, 2002.

25. Singh N, Baby D, Rajguru JP, Patil PB, Thakkannavar SS and Pujari VB: Inflammation and cancer. Ann Afr Med 18: 121-126, 2019.

26. Hanahan D and Weinberg RA: Hallmarks of cancer: The next generation. Cell 144: 646-674, 2011.

27. Clevers $\mathrm{H}$ : At the crossroads of inflammation and cancer. Cell 118: 671-674, 2004

28. Mantovani A: Cancer: Inflammation by remote control. Nature 435: 752-753, 2005.

29. Ha H, Debnath B and Neamati N: Role of the CXCL8-CXCR1/2 axis in cancer and inflammatory diseases. Theranostics 7: 1543-1588, 2017.

30. Todorović-Raković $\mathrm{N}$ and Milovanović J: Interleukin-8 in breast cancer progression. J Interferon Cytokine Res 33: 563-570, 2013.

31. Brandolini L, Bertini R, Bizzarri C, Sergi R, Caselli G, Zhou D, Locati M and Sozzani S: IL-1 beta primes IL-8-activated human neutrophils for elastase release, phospholipase D activity, and calcium flux. J Leukoc Biol 59: 427-434, 1996.

32. Tappia PS, Dent MR and Dhalla NS: Oxidative stress and redox regulation of phospholipase D in myocardial disease. Free Radic Biol Med 41: 349-361, 2006.

33. Li A, Dubey S, Varney ML, Dave BJ and Singh RK: IL-8 directly enhanced endothelial cell survival, proliferation, and matrix metalloproteinases production and regulated angiogenesis. J Immunol 170: 3369-3376, 2003.

34. Khurram SA, Bingle L, McCabe BM, Farthing PM and Whawell SA: The chemokine receptors CXCR1 and CXCR2 regulate oral cancer cell behaviour. J Oral Pathol Med 43: 667-674, 2014. 
35. Siesser PM and Hanks SK: The signaling and biological implications of FAK overexpression in cancer. Clin Cancer Res 12: 3233-3237, 2006.

36. Kopetz S, Shah AN and Gallick GE: Src continues aging: Current and future clinical directions. Clin Cancer Res 13: 7232-7236, 2007.

37. Bournazou E and Bromberg J: Targeting the tumor microenvironment: JAK-STAT3 signaling. JAKSTAT 2: e23828, 2013.

38. Walter M, Liang S, Ghosh S, Hornsby PJ and Li R: Interleukin 6 secreted from adipose stromal cells promotes migration and invasion of breast cancer cells. Oncogene 28: 2745-2755, 2009.

39. Kurosaka M and Machida S: Interleukin-6-induced satellite cell proliferation is regulated by induction of the JAK2/STAT3 signalling pathway through cyclin D1 targeting. Cell Prolif 46: $365-373,2013$

40. Adachi Y, Aoki C, Yoshio-Hoshino N, Takayama K, Curiel DT and Nishimoto N: Interleukin-6 induces both cell growth and VEGF production in malignant mesotheliomas. Int J Cancer 119: 1303-1311, 2006

41. Rokavec M, Öner MG and Hermeking H: Inflammation-induced epigenetic switches in cancer. Cell Mol Life Sci 73: 23-39, 2016.

42. Chang Q, Bournazou E, Sansone P, Berishaj M, Gao SP, Daly L, Wels J, Theilen T, Granitto S, Zhang X, et al: The IL-6/JAK/Stat3 feed-forward loop drives tumorigenesis and metastasis. Neoplasia 15: 848-862, 2013.

43. O-Charoenrat P, Rhys-Evans $\mathrm{PH}$ and Eccles SA: Expression of matrix metalloproteinases and their inhibitors correlates with invasion and metastasis in squamous cell carcinoma of the head and neck. Arch Otolaryngol Head Neck Surg 127: 813-820, 2001

44. Patterson ML, Atkinson SJ, Knäuper V and Murphy G: Specific collagenolysis by gelatinase A, MMP-2, is determined by the hemopexin domain and not the fibronectin-like domain. FEBS Lett 503: 158-162, 2001.

45. Georgescu EF, Mogoantă SŞ, Costache A, Pârvănescu V, Totolici BD, Pătrascu S and Stănescu C: The assessment of matrix metalloproteinase-9 expression and angiogenesis in colorectal cancer. Rom J Morphol Embryol 56: 1137-1144, 2015.

46. Maciejczyk M, Pietrzykowska A, Zalewska A, Knaś M and Daniszewska I: The significance of matrix metalloproteinases in oral diseases. Adv Clin Exp Med 25: 383-390, 2016.

47. Su CW, Lin CW, Yang WE and Yang SF: TIMP-3 as a therapeutic target for cancer. Ther Adv Med Oncol 11: 1758835919864247, 2019.
48. Jiang Y, Goldberg ID and Shi YE: Complex roles of tissue inhibitors of metalloproteinases in cancer. Oncogene 21: 2245-2252, 2002.

49. Lu YC, Chang JT, Liao CT, Kang CJ, Huang SF, Chen IH, Huang CC, Huang YC, Chen WH, Tsai CY, et al: OncomiR-196 promotes an invasive phenotype in oral cancer through the NME4-JNK-TIMP1-MMP signaling pathway. Mol Cancer 13: 218, 2014.

50. St John MA, Li Y, Zhou X, Denny P, Ho CM, Montemagno C, Shi W, Qi F, Wu B, Sinha U, et al: Interleukin 6 and interleukin 8 as potential biomarkers for oral cavity and oropharyngeal squamous cell carcinoma. Arch Otolaryngol Head Neck Surg 130: 929-935, 2004.

51. SahebJamee M, Eslami M, AtarbashiMoghadam F and Sarafnejad A: Salivary concentration of TNFalpha, IL1 alpha, IL6, and IL8 in oral squamous cell carcinoma. Med Oral Patol Oral Cir Bucal 13: E292-E295, 2008.

52. Punyani SR and Sathawane RS: Salivary level of interleukin-8 in oral precancer and oral squamous cell carcinoma. Clin Oral Investig 17: 517-524, 2013

53. Lotfi A, Shahidi N, Bayazian G, AbdollahiFakhim S, Estakhri R, Esfahani A and Notash R: Serum level of interleukin-6 in patients with oral tongue squamous cell carcinoma. Iran J Otorhinolaryngol 27: 207-211, 2015.

54. Rezaei F, Mozaffari HR, Tavasoli J, Zavattaro E, Imani MM and Sadeghi M: Evaluation of serum and salivary interleukin-6 and interleukin-8 levels in oral squamous cell carcinoma patients: Systematic review and meta-analysis. J Interferon Cytokine Res 39: 727-739, 2019

55. Berghi NO, Dumitru M, Vrinceanu D, Ciuluvica RC, Simioniuc-Petrescu A, Caragheorgheopol R, Tucureanu C, Cornateanu RS and Giurcaneanu C: Relationship between chemokines and $\mathrm{T}$ lymphocytes in the context of respiratory allergies (Review). Exp Ther Med 20: 2352-2360, 2020.

(7) $\Theta$ This work is licensed under a Creative Commons Attribution-NonCommercial-NoDerivatives 4.0 International (CC BY-NC-ND 4.0) License. 